\title{
The CD274/TIGIT- and immune microenvironment- combined mutiCox risk model for predicting the overall survival of metastatic PRAD patients: a potential strategy for clinical application
}

hao zheng ( $\sim$ littlestare180710@126.com )

Changhai Hospital https://orcid.org/0000-0003-4378-6560

Chen Ye

Changhai Hospital

Guangan Xiao

Changhai Hospital

Chen Kong

new jianwan city community

Jin Ji

Changhai Hospital

Yuangui Chen

Changhai Hospital

Shengfei Qin

Changhai Hospital

caiguo huang

Second Military Medical University

zhi cao

Changhai Hospital

\section{Research Article}

Keywords: metastatic PRAD patients, immune checkpoints, tumor-infiltrating lymphocytes, overall survival

Posted Date: February 11th, 2022

DOI: https://doi.org/10.21203/rs.3.rs-1322453/v1

License: (a) This work is licensed under a Creative Commons Attribution 4.0 International License. Read Full License 


\section{Abstract}

Purpose: The five-year overall survival rate in metastatic prostate cancer (M-PRAD) is extremely low. In this study, we constructed a more accurate and reliable prediction model for the overall survival (OS) of M-PRAD by combining the expression of PD-L1 and TIGIT with the immune microenvironment, providing a more accurate decision-making basis for clinical treatment.

Patients and Methods: This study, 200 in-house patients with PRAD who underwent prostatectomy between January 2002 and December 2017 were included. In addition, 496 PRAD patients from TCGA database and 102 PRAD patients from SU2C-PCF Dream Team Dataset were included. The immunohistochemical slices in this test were assessed by two pathologists at least 10 years of experience. Kaplan-meier survival curve, ROC curve and multivariate (muti) Cox regression analysis were calculated using $\mathrm{R}$ language.

Results: PD-L1 was down-regulated while TIGIT was up-regulated expression in PRAD tumor tissues compared with adjacent normal tissues, and differentially expressed PD-L1 and TIGIT can be used to assess OS in patients with M-PRAD. Univariate survival analysis showed that tumor-associated lymphocytes (TILs), B cells, dendritic cells (DCs), MHC_class_I, And T_cell_co-stimulation cannot be used as a clinical indicator to evaluate the OS of M-PRAD patients. However, the mutiCox model of PD-L1 and TIGIT combined with these six immune indicators were more accurately and reliably to predict the OS of M-PRAD.

Conclusion: These data indicate that the prediction model developed in this study helps timely clinical decisions in M-PRAD patients.

\section{Introduction}

Prostate cancer (PRAD) is the most commonly diagnosed non-skin cancer type and the leading cause of cancer-related death in men [1]. Progression of metastatic disease status is a major factor in most PRAD deaths and $98 \%$ of patients with metastatic prostate cancer (M-PRAD) are expected to have an overall survival (OS) of less than five years [2;3]. Therefore, the development of a model to predict the OS of MPRAD patients is conducive to timely treatment.

Tumor cells promote tumor progression, to some extent, by activating immune checkpoint pathways that inhibit anti-tumor immune responses [4]. In recent years, PD-L1 (B7-H1, CD274, a ligand of PD-1 and belongs to the protein B7 family) and T cell immunoglobulin and ITIM domain (TIGIT, an inhibitory receptor expressed on lymphocytes) has attracted much attention as the targets for tumor immunotherapy [5; 6]. PD-L1 was usually up-regulated in solid tumors, hemangioma and antigenpresenting cells [7], and the tumor immune escape is mediated by abnormally high expression of PD-L1 [8]. TIGIT, a negative regulator of cytotoxic lymphocytes, is usually increased expressed on NK cells, T cells and various malignancies, and its mechanism of limiting antitumor response plays an important 
role in tumor immune escape [5]. Therefore, we intend to develop a model to predict the OS of patients with M-PRAD based on the expression of PD-L1 and TIGIT.

In this study, we performed immunohistochemical (IHC) detection on PRAD tumors and paired normal tissues, and combined The Cancer Genome Atlas (TCGA) -PRAD and SU2C-PCF Dream Team dataset [9] to evaluate the diagnostic utility of PD-L1 and TIGIT for PRAD patients. We used PRAD queues (including non-M-PRAD and M-PRAD) initially, and selected M-PRAD cases intentionally to evaluate the diagnostic value of PD-L1 and TIGIT for predicting the OS of PRAD. In addition, in the mutiCox risk model established based on the expression of PD-L1 and TIGIT, we found that PD-L1 and TIGIT have a substantial impact on the immune microenvironment of the tumor tissues, and the PD-L1/TIGIT- and immune microenvironment-combined mutiCox risk model revealed a significant difference in the OS of MPRAD patients. Toward this end, we have developed a new diagnostic model for predicting OS in M-PRAD patients, which may be suitable for clinical applications.

\section{Methods}

\section{Patients and Samples}

All the 200 tumor tissues and paired normal tissues, from the patients treated with radical prostatectomy for PRAD who has not gained therapy like preoperative chemotherapy or radiotherapy before, were obtained from specimen repository of Changhai Hospital from January 2002 and December 2017. This experiment got written informed and consent of all patients. All procedures performed in studies involving human participants were in accordance with the ethical standards of the Research Ethics Committee of Changhai hospital. This study achieved the permission from the ethics committee of Changhai Hospital.

\section{IHC detection}

After cut into 4- $\mu \mathrm{m}$ sections, all the tissues were deparaffinized and treated with EDTA (pH 9.0) to antigen retrieval in a microwave for 20 min. Then, Autostainer Link 48 machine (Dako, Denmark A/S, Denmark) was performed for staining. Subsequently, primary antibodies TIGIT (rabbit monoclonal, 1:100 dilution, BLR047F, Abcam) and CD274 (rabbit monoclonal, 1:200 dilution; ab205921, Abcam) were added to the sections, while PBS buffer was used as a blank control instead of the antibody. EnVision Flex Kit was used as the second antibody (Dako, Denmark A/S, Denmark). All cases were examined by two senior pathologists to validate initial scores. Percentage of positively stained cells and staining scores were used to assess the IHC results and the detail referred to the previous article [10; 11]. Gene-Score = (percentage of cells of weak intensity $\times 1)+($ percentage of cells of moderate intensity $\times 2$ ) + percentage of cells of strong intensity $\times 3)$.

\section{Bioinformation analysis}

The RNA-sequencing (RNA-seq) data was obtained from The Cancer Genome Atlas (TCGA) (https://portal.gdc.cancer.gov/) and Genotype-Tissue Expression (GTEx, 
https://commonfund.nih.gov/GTEx/) database, and remove batch effects using the COMBAT function of the R package "SVA". The SU2C-PCF Dream Team dataset was provided by Sawyers CL et.al. [9]. The Kaplan-Meier Survival Curve, Roc Curve and Muticox Model analysis were conducted using the R package "ggPlot2". The infiltration of immune cells was calculated via single sample gene set enrichment analysis (sSGSEA) using the R package or via the cibersortx assays base on website tools (https://cibersortx.stanford.edu/).

\section{Statistical analysis}

Data analysis was performed using IBM ${ }^{8} S P S S \AA$, version 24.0 (IBM Corp., Armonk, NY, USA). The data was expressed in a mean \pm standard deviation. Kruskal-Wallis test was used to compare data among multiple groups. Wilcox test was used to compare data betwenn two groups. $\mathrm{P}<0.05$ was considered significant.

\section{Results}

\section{The expression level and prognostic value of TIGIT and CD274 mRNA in pan-cancer}

TIGIT was up-regulated expression in tumor tissues compared to adjacent normal tissues in pan-cancer (Figure 1A). However, CD274 was up-regulated or down-regulated expression in tumor tissues compared to adjacent normal tissues in different tumor types (Figure 1B). In addition, uniCox analysis showed that differentially expressed TIGIT (Figure 1C) or CD274 (Figure 1D) can be used to evaluate disease-free survival (DFS), disease-specific survival (DSS), overall survival (OS) and progression-free survival (PFS) in multiple tumor types. Compared with adjacent normal tissues, TIGIT (Figure 1A) was up-regulated expression while CD274 (Figure 1B) was down-regulated expression in tumor tissues, and the differentially expressed TIGIT (Figure 1C) or CD274 (Figure 1D) can be used as the risk factors for assessing the prognosis of PRAD patients.

\section{Detection of the mutation and protein expression levels of CD274 and TIGIT in PRAD}

The mutation rates of CD274 and TIGIT was both 1.3\% in 7161 PRAD samples according to cbioportal tool (https://www.cbioportal.org/) (Figure 2A). In our in-house cohort, the TIGIT was found up-regulated expression while CD274 was found down-regulated expression in non-metastatic tumor tissues $(n=67)$ and metastatic tumor tissues $(n=133)$ compared to paired adjacent normal tissues $(n=200)$ via IHC detection at protein level (Figure 2B), which was consistent with mRNA level in TCGA dataset (Figure 1A and B). Differentially expressed CD274 and TIGIT, rather than mutations, may be related to prognosis of PRAD patients. 


\section{Differential expressed CD274 and TIGIT were only suitable for predicting overall survival in the metastatic cohort of PRAD patients}

In the cohort of PRAD patients with or without metastatic tumor ( $n=495$, TCGA), the differential expressed CD274 and TIGIT mRNA had no significant effect on OS in PRAD patients (Supplementary Figure 1), and this result was confirmed in in-house cohort of non-metastatic tumor tissues $(n=98)$ and metastatic tumor tissues $(n=67)$ at protein levels (Supplementary Figure 2). However, the low expression of CD274 and the high expression of TIGIT predict poorer OS in M-PRAD patients at mRNA in the metastatic cohort of SU2C-PCF Dream Team ( $n=102)$ (Figure 3 ) and at protein levels in the metastatic cohort of in-house MPRAD patients $(n=67)$ (Figure 4).

\section{CD274 and TIGIT are closely related to the immune microenvironment of metastatic PRAD patients}

We constructed risk models for CD274 and TIGIT by mutiCox. Compared with the low-risk group, the OS of M-PRAD patients in the high-risk group were substantially reduced (Supplementary Figure 3). Based on the Cibersort (Supplementary Figure 4) and SsGSEA algorithms (Figure 5), we further evaluate the tumor immune microenvironment of M-PRAD patients. Due to the existence of many missing values in the results obtained by Cibersortx algorithm, no difference in immune microenvironment was found between risk-low and risk-high groups (Supplementary Figure 4). The results of sSGSEA algorithms showed that tumor-associated lymphocytes (TILs), B cells, dendritic cells (DCs), MHC_class_I, and T_cell_costimulation sub-groups were substantially increased, while the neutrophils were substantially reduced in the risk-high group compared with the risk-low group (Figure 5).

\section{The CD274/TIGIT- and immune microenvironment- combined mutiCox risk model for predicting OS in metastatic PRAD patients}

Although B_cells, DCs, MHC_class_I, Neutrophils, T_cell_co-stimulation, and TILs are ineffective indicators for predicting OS in M-PRAD patients in single factor level (Supplementary Figure 5). However, the mutiCox risk model showed that the M-PRAD patients in the risk-high group had worse OS than the risklow group, according to the mutiCox risk model constructed from these six immune cells (Figure 6). In addition, we further combined CD274, TIGIT and the six immune cells to construct the mutiCox risk model, and the results showed substantial differences in OS between the risk-low and risk-high groups in the M-PRAD patients (Figure 7).

\section{Discussion}

Although PD-L1 was highly expressed in small-cell lung cancer [12], urinary tract epithelial carcinoma [13] and head and neck carcinoma [14], and these tumor types had shown clinical efficacy in anti-PD-L1 
antibody treatment. However, PD-L1 was almost unexpressed or low expressed in primary PRAD [15] and high-risk prostate cancer tissues [16]. In addition, no efficacy was shown in the treatment of castrationresistant PRAD with anti-PD-L1 antibody [17]. Based on the cohort of 52 adjacent normal tissues and 499 PRAD tumor tissues provided by TCGA database, the down-regulation of CD274 mRNA was also observed in PRAD tumor tissues. In addition, we performed IHC detection of CD274 protein in 200 adjacent normal tissues, 67 M-PRAD tumor tissues and 133 non-M-PRAD tumor tissues. CD274 was down-regulated expression in both M-PRAD and non-M-PRAD tumor tissues compared to adjacent normal tissues substantially, and the expression of CD274 in M-PRAD tumor tissues was substantially lower than that in non-M-PRAD tumor tissues. Immune checkpoint inhibitors tend to be more effective against tumors with high tumor mutation load, such as melanoma and non-small cell lung cancer, the two tumors with the highest average number of mutations [14]. However, based on 7161 PRAD samples provided by the Cbiopotal database, the TMB of CD274 was only 1.3\%. Therefore, CD274 was under-expressed in patients with PRAD and may be ineffective against PD-L1 inhibition.

The up-regulated expression of TIGIT has been observed in melanoma, breast cancer, gastric cancer and other malignant tumors [5]. However, the expression of TIGIT in prostate cancer has not been characterized. Compared to adjacent normal tissues, TIGIT protein was substantially up-regulated expression in both M-PRAD and non-M-PRAD tumor tissues, while no difference was found between MPRAD and non-M-PRAD tumor tissues via IHC detection, and consistent with TCGA-PRAD cohort at mRNA level. Currently, six anti-TIGIT mAbs have started clinical trials, including tiragomab (MTIG7192A; RG6058), AB154, MK-7684, BMS-986207, ASP8374, and ASP8374 [18]. Compared with PD-L1 inhibitors, anti-TIGIT mAb may be a better choice for PRAD patients.

In both our in-house cohort of CD274/TIGIT protein and TCGA cohort of CD274/TIGIT mRNA shown that the differentially expressed CD274 or TIGIT was not suitable for predicting OS in PRAD patients. However, the down-regulated expression of CD274 or up-regulated expression of TIGIT predicted poorer OS in MPRAD patients in our in-house cohort and SU2C-PCF Dream Team cohort. In humans and mice, CD155 is one of the main ligand of TIGIT, which interacted to inhibit the function of effector T cells and NK cells [18]. In addition, CD274 was the main ligand of PD-1, which interacted to inhibit the function of immune effector cells [19]. Based on the mutiCox risk model of CD274/TIGIT, we divided M-PRAD cohort into riskhigh group and risk-low group. Through ssGSEA analysis, we found that compared with risk-low group, TILs, B Cells, DCs, MHC_class_I, and T_cell_co-stimulation were substantially increased but neutrophils were substantially decreased in risk-high group.

In tumor microenvironment, DCs was generally regarded as necessary for T cell-mediated cancer immunity [20]. Normally, tumor-associated DCs captured tumor antigens and activated tumor-specific T cells in draining lymph nodes [21]. However, considering the inhibitory mechanism within the tumor, DCs are usually insufficient to induce effective tumor immunity and depend on a specific subset of DCs [20]. The activation of tumor antigen-specific T cells was effectively stimuli via tumor-associated CD $103^{+}$DCs and CD11 b+ DCs [22]. However, the release of nitric oxide and arginase I in tumor-associated DCs inhibited the function of tumor-specific T cells [23]. As a carcinogenic driver, STAT3 enhances tumor cell 
proliferation, survival and invasion, and inhibits the differentiation, maturation and function of DCs [24; 25]. The tumor-derived factor Toll-like receptor 2 stimulates the production of autocrine IL-6 and IL-10 in CDC, and promotes DCs dysfunction [25]. Moreover, after TIGIT interacted with CD155, the antigen presentation ability of $\mathrm{CD} 155^{+} \mathrm{DCs}$ decreases, and the secretion of co-stimulatory molecules and proinflammatory cytokines decrease, which in turn inhibits the activation of T cells [5]. The up-regulation of PD-L1 can inhibit the killing of DCs by cytotoxic T lymphocytes and inhibit the anti-tumor response [26]. However, Blocking PD-L1 on dendritic cells promotes the secretion of IL-10 by T cells [27], which impaired the function of DCs [25]. We speculate that DCs in the risk-high group may be immunosuppressive subtypes, and the subtypes of DCs need to be further confirmed.

Generally, tumors can evade T cell responses by down-regulating MHC class I molecules [28]. According to reports, in metastatic uveal melanoma, MHC class I negative has a better prognosis than $\mathrm{MHC}$ class I positive [46]. In the process of colonization of colorectal tumor cells in liver tissue, NK cells kill MHC class I negative tumor cells, thereby selecting MHC class I positive tumor cells for metastasis [29]. The possible explanation for this phenomenon is that the complete loss of MHC class I antigen expression makes cells susceptible to NK cell-mediated killing [30]. In the high-risk group, the high expression of MHC I may be beneficial to the metastasis of PRAD tumor cells.

It is well known that B cells promote $\mathrm{T}$ cell activation and proliferation through antigen presentation [31]. However, studies have shown that suppressor B cells have the ability to maintain immune tolerance and promote the expression of IL-10 [32;33]. In this study, we did not classify the subtypes of B cells. The proportion of inhibitory B cells in the risk-high group may increase, and further verification is needed.

T cell co-signaling receptors can transduce positive (co-stimulatory receptors) or negative (co-inhibitory receptors) regulated TCR signals into T cells. However, there is a big gap in the characterization of the unique functions of each co-stimulatory and co-inhibitory receptor [34]. In this study, we initially found that in the risk-high group, the proportion of T_cell_co-stimulation increased, but its underlying mechanism deserves further exploration.

Neutrophils are a plastic heterogeneous population with tumor-promoting and anti-tumor effects. Studies have shown that neutrophils can promote tumor proliferation by secreting elastase, drive angiogenesis by providing secretion of MMP-9, and promote tumor cell metastasis by degrading extracellular matrix [35]. However, tumor-associated neutrophils inhibited tumor cell metastasis by releasing hydrogen Peroxide $\left(\mathrm{H}_{2} \mathrm{O}_{2}\right)$ that kills disseminated tumor cells [36; 37]. Neutrophils may inhibit the metastasis of PRAD cells by releasing $\mathrm{H} 2 \mathrm{O} 2$ In metastatic PRAD tumor tissues. In this study, the cell proportion of neutrophils was substantially reduced in the high-risk group, prompting the risk of tumor metastasis.

In summary, we further constructed a mutiCox model based on CD274/TIGIT and the above-mentioned immune cells, and the results showed that it has significance clinical value in predicting OS in M-PRAD patients, and has good sensitivity and specificity via ROC analysis. 


\section{Declarations}

\section{ETHICS STATEMENT}

This experiment got written informed and consent of all patients. All procedures performed in studies involving human participants were in accordance with the ethical standards of the Research Ethics Committee of Changhai hospital. This study achieved the permission from the ethics committee of Changhai Hospital.

\section{Competing Interest}

The authors declare no potential conflict of interests.

\section{FUNDING}

This work was supported by the National Natural Science Foundation of China (No. 81472397, 81301861), National Key Basic Research Program of China (973 Program, No. 2012CB518306) and National Key Research and Development Program of China (No.2018YFC0310904).

\section{AUTHOR CONTRIBUTIONS}

CGH and ZC were responsible for the design of this research. CY and GGX were responsible for collecting samples and conducting IHC. All authors participate in the data processing, and manuscript writing.

\section{References}

1. Z. Wu, H. Chen, W. Luo, H. Zhang, G. Li, F. Zeng, and F. Deng, The Landscape of Immune Cells Infiltrating in Prostate Cancer. Frontiers in oncology 10 (2020) 517637.

2. G. Wang, D. Zhao, D.J. Spring, and R.A. DePinho, Genetics and biology of prostate cancer. Genes \& development 32 (2018) 1105-1140.

3. C.M. Tangen, J.R. Faulkner, E.D. Crawford, I.M. Thompson, D. Hirano, M. Eisenberger, and M. Hussain, Ten-year survival in patients with metastatic prostate cancer. Clin Prostate Cancer 2 (2003) 41-5.

4. P. Darvin, S.M. Toor, V. Sasidharan Nair, and E. Elkord, Immune checkpoint inhibitors: recent progress and potential biomarkers. Experimental \& molecular medicine 50 (2018) 1-11.

5. H. Harjunpaa, and C. Guillerey, TIGIT as an emerging immune checkpoint. Clinical and experimental immunology 200 (2020) 108-119.

6. Y. Han, D. Liu, and L. Li, PD-1/PD-L1 pathway: current researches in cancer. American journal of cancer research 10 (2020) 727-742. 
7. S.P. Patel, and R. Kurzrock, PD-L1 Expression as a Predictive Biomarker in Cancer Immunotherapy. Molecular cancer therapeutics 14 (2015) 847-56.

8. Y. Jiang, M. Chen, H. Nie, and Y. Yuan, PD-1 and PD-L1 in cancer immunotherapy: clinical implications and future considerations. Hum Vaccin Immunother 15 (2019) 1111-1122.

9. W. Abida, J. Cyrta, G. Heller, D. Prandi, J. Armenia, I. Coleman, M. Cieslik, M. Benelli, D. Robinson, E.M. Van Allen, A. Sboner, T. Fedrizzi, J.M. Mosquera, B.D. Robinson, N. De Sarkar, L.P. Kunju, S. Tomlins, Y.M. Wu, D. Nava Rodrigues, M. Loda, A. Gopalan, V.E. Reuter, C.C. Pritchard, J. Mateo, D. Bianchini, S. Miranda, S. Carreira, P. Rescigno, J. Filipenko, J. Vinson, R.B. Montgomery, H. Beltran, E.I. Heath, H.I. Scher, P.W. Kantoff, M.E. Taplin, N. Schultz, J.S. deBono, F. Demichelis, P.S. Nelson, M.A. Rubin, A.M. Chinnaiyan, and C.L. Sawyers, Genomic correlates of clinical outcome in advanced prostate cancer. Proceedings of the National Academy of Sciences of the United States of America 116 (2019) 11428-11436.

10. W. Yeo, S.L. Chan, F.K. Mo, C.M. Chu, J.W. Hui, J.H. Tong, A.W. Chan, J. Koh, E.P. Hui, H. Loong, K. Lee, L. Li, B. Ma, K.F. To, and S.C. Yu, Phase I/II study of temsirolimus for patients with unresectable Hepatocellular Carcinoma (HCC)- a correlative study to explore potential biomarkers for response. BMC cancer 15 (2015) 395.

11. H.A. Azim, Jr., F.A. Peccatori, S. Brohee, D. Branstetter, S. Loi, G. Viale, M. Piccart, W.C. Dougall, G. Pruneri, and C. Sotiriou, RANK-ligand (RANKL) expression in young breast cancer patients and during pregnancy. Breast Cancer Res 17 (2015) 24.

12. L. Fehrenbacher, A. Spira, M. Ballinger, M. Kowanetz, J. Vansteenkiste, J. Mazieres, K. Park, D. Smith, A. Artal-Cortes, C. Lewanski, F. Braiteh, D. Waterkamp, P. He, W. Zou, D.S. Chen, J. Yi, A. Sandler, A. Rittmeyer, and P.S. Group, Atezolizumab versus docetaxel for patients with previously treated nonsmall-cell lung cancer (POPLAR): a multicentre, open-label, phase 2 randomised controlled trial. Lancet 387 (2016) 1837-46.

13. T. Powles, P.H. O'Donnell, C. Massard, H.T. Arkenau, T.W. Friedlander, C.J. Hoimes, J.L. Lee, M. Ong, S.S. Sridhar, N.J. Vogelzang, M.N. Fishman, J. Zhang, S. Srinivas, J. Parikh, J. Antal, X. Jin, A.K. Gupta, Y. Ben, and N.M. Hahn, Efficacy and Safety of Durvalumab in Locally Advanced or Metastatic Urothelial Carcinoma: Updated Results From a Phase 1/2 Open-label Study. JAMA Oncol 3 (2017) e172411.

14. P. Isaacsson Velho, and E.S. Antonarakis, PD-1/PD-L1 pathway inhibitors in advanced prostate cancer. Expert Rev Clin Pharmacol 11 (2018) 475-486.

15. A.M. Martin, T.R. Nirschl, C.J. Nirschl, B.J. Francica, C.M. Kochel, A. van Bokhoven, A.K. Meeker, M.S. Lucia, R.A. Anders, A.M. DeMarzo, and C.G. Drake, Paucity of PD-L1 expression in prostate cancer: innate and adaptive immune resistance. Prostate Cancer Prostatic Dis 18 (2015) 325-32.

16. W. Baas, S. Gershburg, D. Dynda, K. Delfino, K. Robinson, D. Nie, J.H. Yearley, and S. Alanee, Immune Characterization of the Programmed Death Receptor Pathway in High Risk Prostate Cancer. Clin Genitourin Cancer 15 (2017) 577-581. 
17. S.L. Topalian, F.S. Hodi, J.R. Brahmer, S.N. Gettinger, D.C. Smith, D.F. McDermott, J.D. Powderly, R.D. Carvajal, J.A. Sosman, M.B. Atkins, P.D. Leming, D.R. Spigel, S.J. Antonia, L. Horn, C.G. Drake, D.M. Pardoll, L. Chen, W.H. Sharfman, R.A. Anders, J.M. Taube, T.L. McMiller, H. Xu, A.J. Korman, M. JureKunkel, S. Agrawal, D. McDonald, G.D. Kollia, A. Gupta, J.M. Wigginton, and M. Sznol, Safety, activity, and immune correlates of anti-PD-1 antibody in cancer. N Engl J Med 366 (2012) 2443-54.

18. X. Yu, K. Harden, L.C. Gonzalez, M. Francesco, E. Chiang, B. Irving, I. Tom, S. Ivelja, C.J. Refino, H. Clark, D. Eaton, and J.L. Grogan, The surface protein TIGIT suppresses T cell activation by promoting the generation of mature immunoregulatory dendritic cells. Nat Immunol 10 (2009) 48-57.

19. F. Schutz, S. Stefanovic, L. Mayer, A. von Au, C. Domschke, and C. Sohn, PD-1/PD-L1 Pathway in Breast Cancer. Oncology research and treatment 40 (2017) 294-297.

20. A. Gardner, and B. Ruffell, Dendritic Cells and Cancer Immunity. Trends Immunol 37 (2016) $855-865$.

21. O.J. Finn, Cancer immunology. N Engl J Med 358 (2008) 2704-15.

22. M.L. Broz, M. Binnewies, B. Boldajipour, A.E. Nelson, J.L. Pollack, D.J. Erle, A. Barczak, M.D. Rosenblum, A. Daud, D.L. Barber, S. Amigorena, L.J. Van't Veer, A.I. Sperling, D.M. Wolf, and M.F. Krummel, Dissecting the tumor myeloid compartment reveals rare activating antigen-presenting cells critical for T cell immunity. Cancer cell 26 (2014) 638-52.

23. D.H. Munn, and V. Bronte, Immune suppressive mechanisms in the tumor microenvironment. Curr Opin Immunol 39 (2016) 1-6.

24. Y. Nefedova, M. Huang, S. Kusmartsev, R. Bhattacharya, P. Cheng, R. Salup, R. Jove, and D. Gabrilovich, Hyperactivation of STAT3 is involved in abnormal differentiation of dendritic cells in cancer. Journal of immunology 172 (2004) 464-74.

25. H. Yu, M. Kortylewski, and D. Pardoll, Crosstalk between cancer and immune cells: role of STAT3 in the tumour microenvironment. Nature reviews. Immunology 7 (2007) 41-51.

26. H. Tryphonas, L. O'Grady, S. Hayward, and Z. Zawidzka, Enumeration of lymphocyte subsets in peripheral blood of rhesus monkeys prior to and following monocyte removal using a modified indirect avidin-biotin immunoperoxidase technique. Vet Immunol Immunopathol 19 (1988) 119-26.

27. J.A. Brown, D.M. Dorfman, F.R. Ma, E.L. Sullivan, O. Munoz, C.R. Wood, E.A. Greenfield, and G.J. Freeman, Blockade of programmed death-1 ligands on dendritic cells enhances $\mathrm{T}$ cell activation and cytokine production. Journal of immunology 170 (2003) 1257-66.

28. F. Garrido, and N. Aptsiauri, Cancer immune escape: MHC expression in primary tumours versus metastases. Immunology 158 (2019) 255-266.

29. M. Kloor, S. Michel, and M. von Knebel Doeberitz, Immune evasion of microsatellite unstable colorectal cancers. International journal of cancer 127 (2010) 1001-10.

30. A.G. Menon, H. Morreau, R.A. Tollenaar, E. Alphenaar, M. Van Puijenbroek, H. Putter, C.M. JanssenVan Rhijn, C.J. Van De Velde, G.J. Fleuren, and P.J. Kuppen, Down-regulation of HLA-A expression correlates with a better prognosis in colorectal cancer patients. Lab Invest 82 (2002) 1725-33.

31. T.W. LeBien, and T.F. Tedder, B lymphocytes: how they develop and function. Blood 112 (2008) 157080 . 
32. E.C. Rosser, and C. Mauri, Regulatory B cells: origin, phenotype, and function. Immunity 42 (2015) 607-12.

33. Y. Iwata, T. Matsushita, M. Horikawa, D.J. Dilillo, K. Yanaba, G.M. Venturi, P.M. Szabolcs, S.H.

Bernstein, C.M. Magro, A.D. Williams, R.P. Hall, E.W. St Clair, and T.F. Tedder, Characterization of a rare IL-10-competent B-cell subset in humans that parallels mouse regulatory B10 cells. Blood 117 (2011) 530-41.

34. L. Chen, and D.B. Flies, Molecular mechanisms of T cell co-stimulation and co-inhibition. Nature reviews. Immunology 13 (2013) 227-42.

35. R. Grecian, M.K.B. Whyte, and S.R. Walmsley, The role of neutrophils in cancer. British medical bulletin 128 (2018) 5-14.

36. Z. Granot, E. Henke, E.A. Comen, T.A. King, L. Norton, and R. Benezra, Tumor entrained neutrophils inhibit seeding in the premetastatic lung. Cancer cell 20 (2011) 300-14.

37. M. Gershkovitz, Y. Caspi, T. Fainsod-Levi, B. Katz, J. Michaeli, S. Khawaled, S. Lev, L. Polyansky, M.E. Shaul, R.V. Sionov, L. Cohen-Daniel, R.I. Aqeilan, Y.D. Shaul, Y. Mori, R. Karni, Z.G. Fridlender, A.M. Binshtok, and Z. Granot, TRPM2 Mediates Neutrophil Killing of Disseminated Tumor Cells. Cancer research 78 (2018) 2680-2690.

\section{Figures}

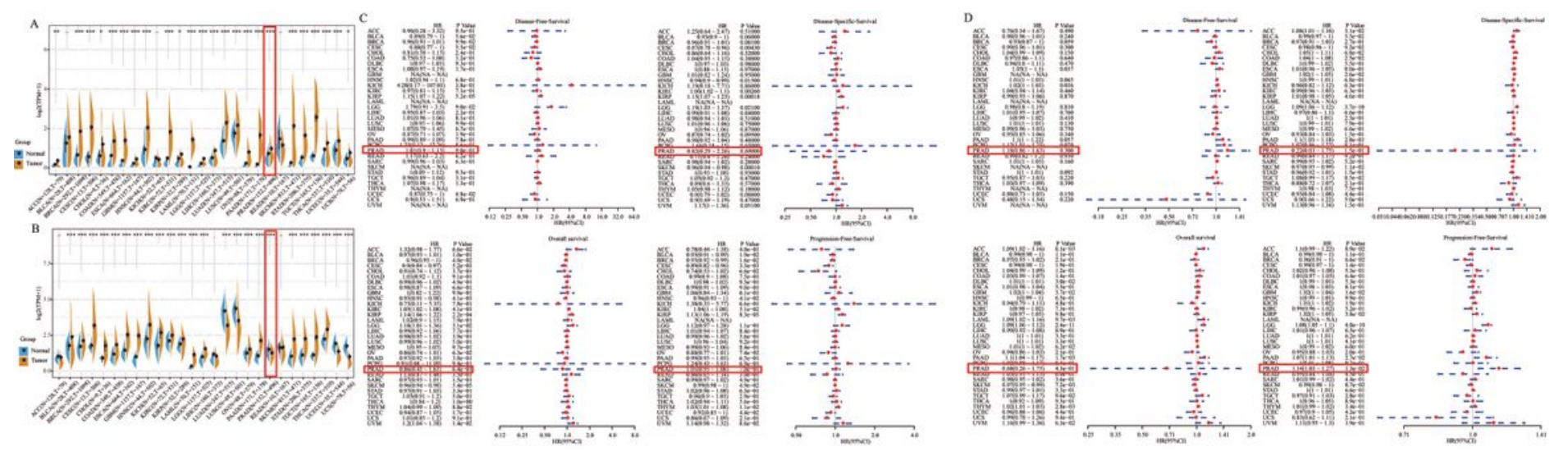

\section{Figure 1}

The TIGIT or CD274 was differentially expressed in pan-cancer, and associated with prognosis of PRAD patients. The mRNA expression of TIGIT (A) and CD274 (B) in pan-cancer, and the disease-free survival (DFS), disease-specific survival (DSS), overall survival (OS) and progression-free survival (PFS) in multiple tumor types were provided by TCGA dataset. Note: ${ }^{\star} P<0.05$, ${ }^{\star \star} P<0.01, \star \star \star * P<0.0001$.

\section{Figure 2}


The mutation rates and protein expression of TIGIT or CD274 was detected in PRAD. (A) The mutation rates of TIGIT or CD274 was obtained from cbioportal tool (https://www.cbioportal.org/). (B) The protein expression of TIGIT or CD274 was detected via IHC detection. Note: ${ }^{*} P<0.05,{ }^{*} P<0.01,{ }^{* \star *} P<0.0001$, ns represents no significance.
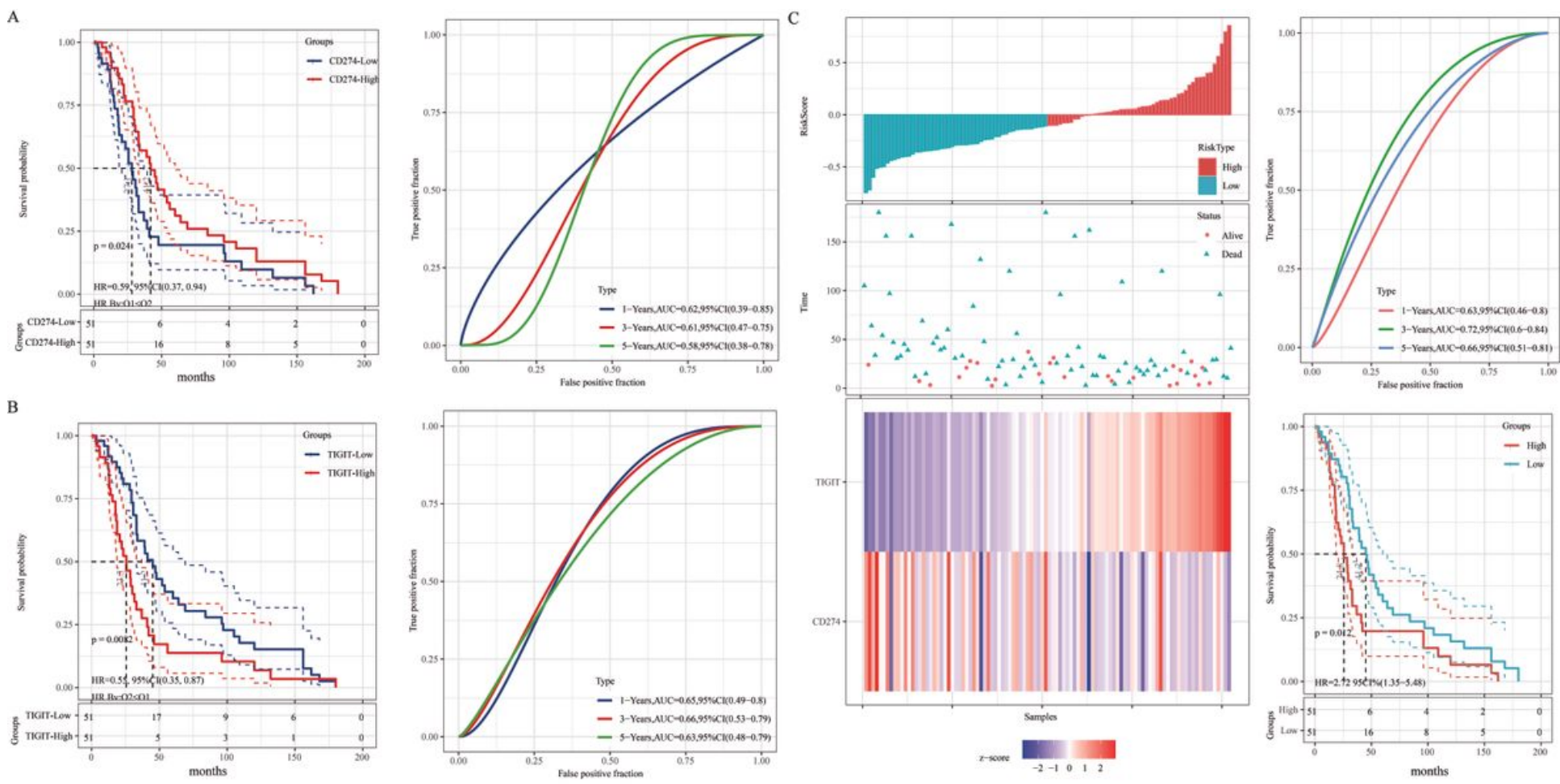

Figure 3

Up-regulated TIGIT or down-regulated CD274 predicted poor prognosis in M-PRAD patients at mRNA level via the SU2C-PCF Dream Team dataset. The up-regulated TIGIT (A) and down-regulated CD274 (B) predicted poor prognosis in M-PRAD patients via Kaplan-Meier survival curve. (C) The down-regulated CD274 combined with the up-regulated TIGIT has better clinical value in predicting overall survival in MPRAD patients via mutiCox model. 

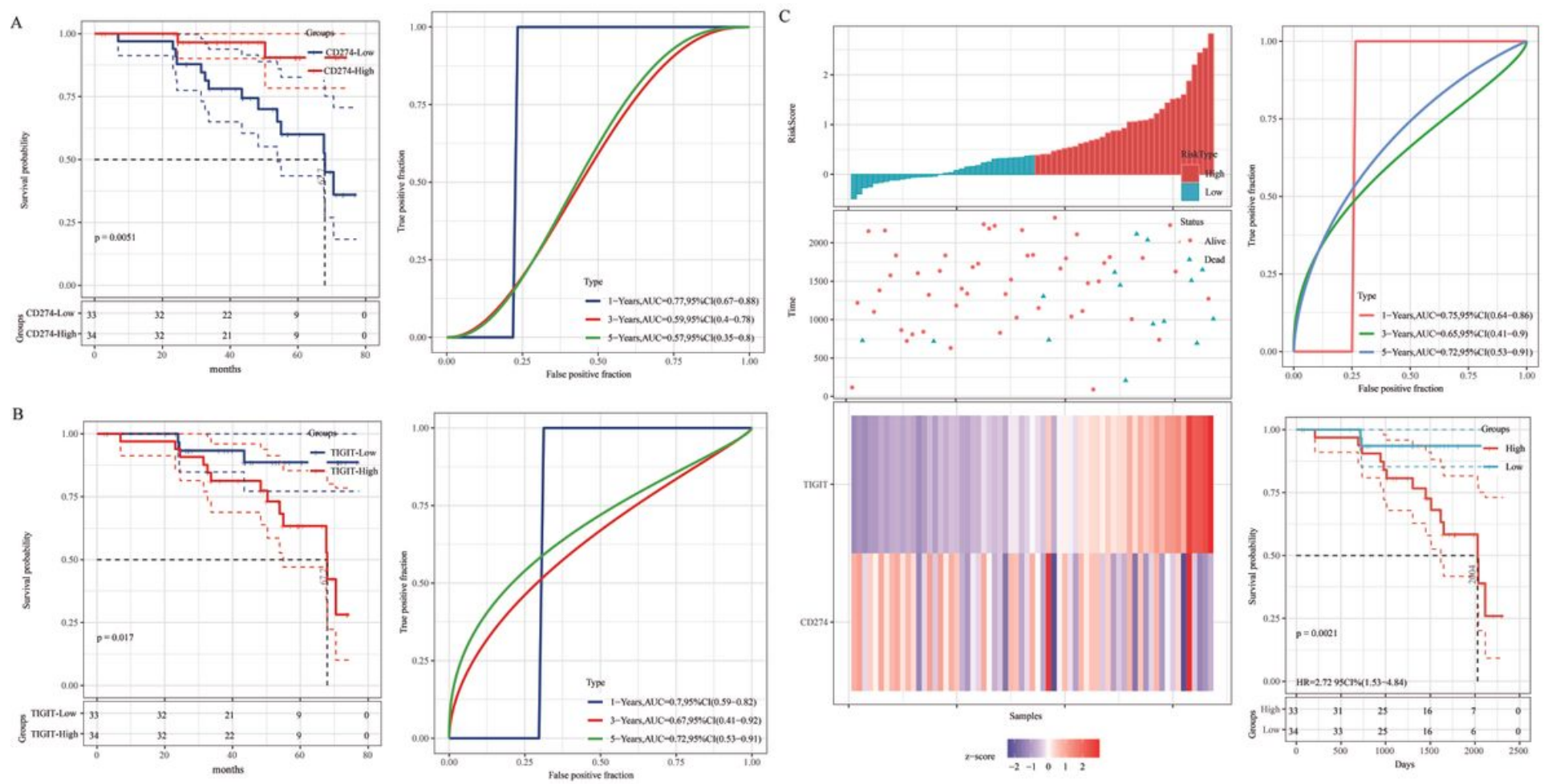

Figure 4

Up-regulated TIGIT or down-regulated CD274 protein expression predicted poor prognosis in M-PRAD patients via IHC detection. The up-regulated TIGIT (A) and down-regulated CD274 (B) protein expression predicted poor OS in M-PRAD patients via Kaplan-Meier survival curve. (C) The down-regulated CD274 protein expression combined with the up-regulated TIGIT protein expression has better clinical value in predicting OS in M-PRAD patients via mutiCox model.

\section{Figure 5}

The infiltration of immune cells in tumor tissues of M-PRAD patients was evaluated based on ssGSEA algorithms. 

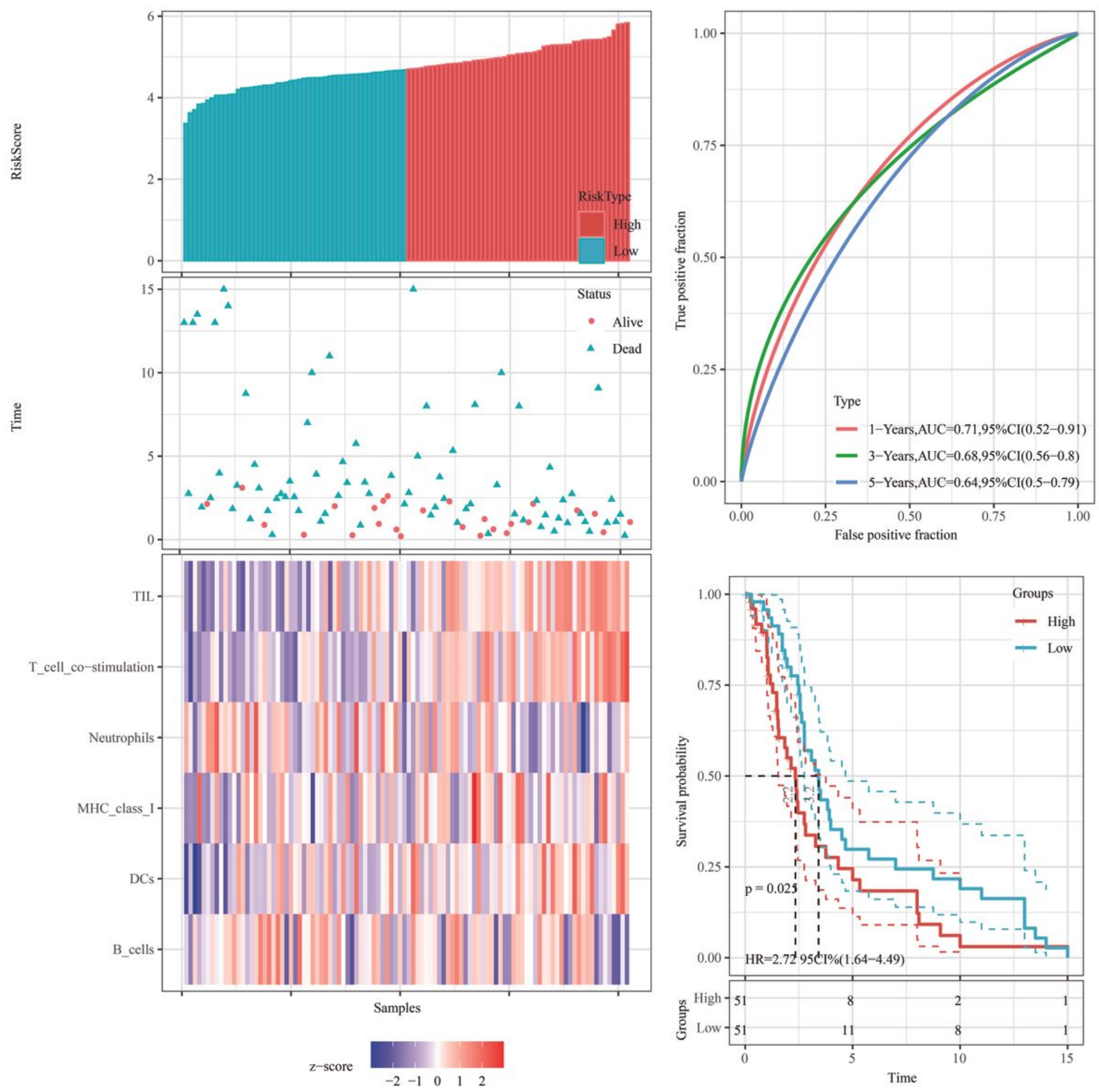

Figure 6

The mutiCox model based on B_cells, DCs, MHC_class_l, Neutrophils, T_cell_co-stimulation, and TILs has good clinical value in predicting the OS of M-PRAD patients. 

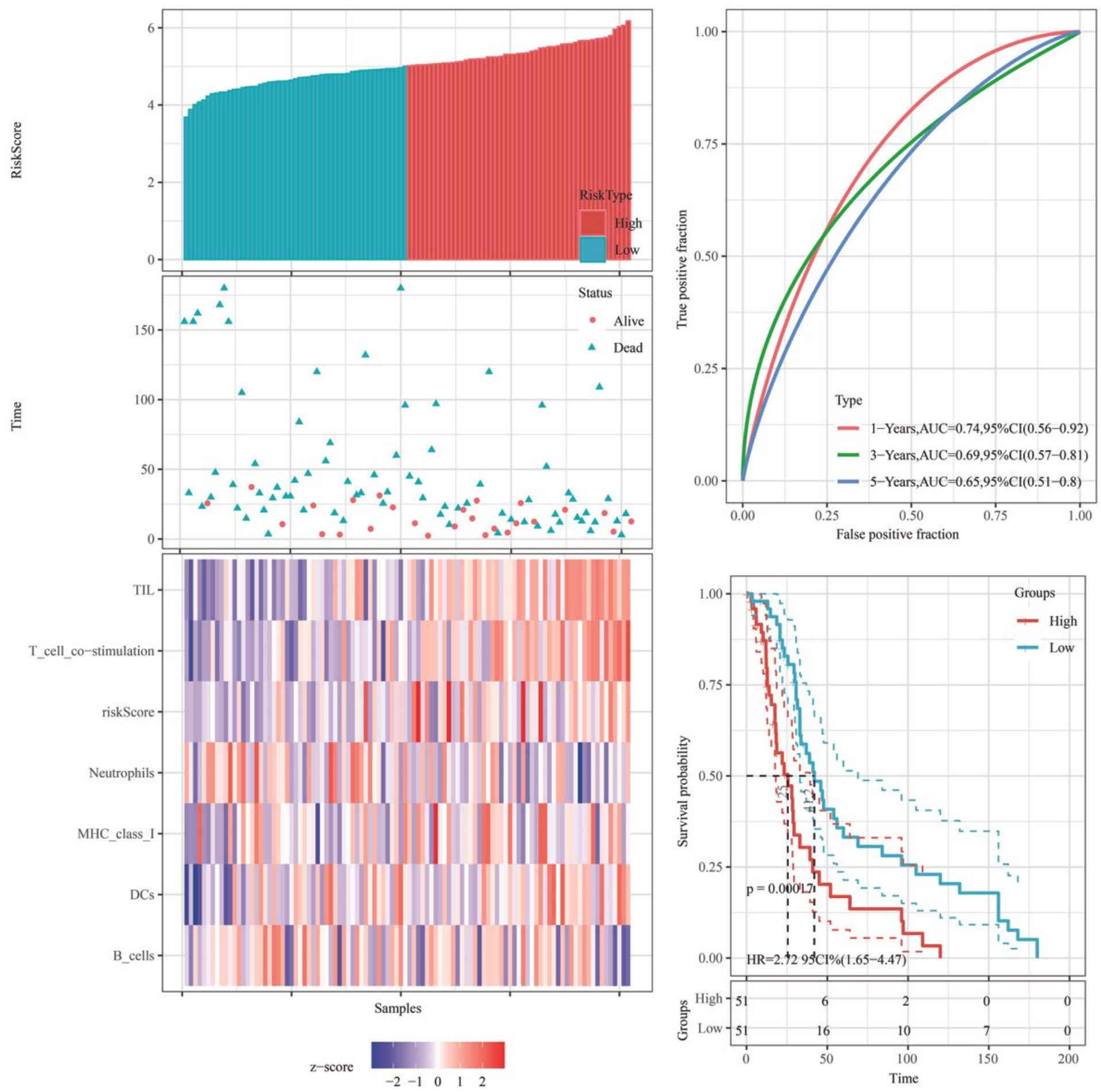

Figure 7

The CD274/TIGIT and immune environment combined mutiCox model has good clinical value in predicting the OS of M-PRAD patients.

\section{Supplementary Files}

This is a list of supplementary files associated with this preprint. Click to download. 
- si.docx

Page 16/16 